\title{
Efficient charging pad for unmanned aerial vehicle based on direct contact
}

\begin{abstract}
Recently there has been significant interest in the development of autonomously Unmanned Aerial Vehicles (UAVs), especially the rotor-based robots, which are highly maneuverable and can vertically take-off and land. However, the discharge characteristics and charge storage capacity limitations of their lithium-ion battery can restrict their flight time endurance. The utilization of an automatic drone charging station is therefore desirable for these robots. This paper proposes a fully automatic contact-based charging station for UAVs to recharge UAV's and thereby solve the UAV's flight endurance limitations. The ground station comprises square-shaped copper plates of consecutively polarized positively and negatively in the form of a chess board with specific dimensions to ensure electrical contact when landing. The design methodology employed with the charging station accounts for the variations of the orientation of the UAV after landing on the platform. Furthermore, this innovation employs an autonomous recharging process after touchdown. Subsequently, this technology relaxes usual flight time constraints and improves overall mission times. The UAV equipped with a suitable hardware circuit the onboard circuit consists of six bridge diode rectifiers to modulate the polarity of the four UAV's contact terminals that allows autonomous recharge regardless of the yaw angle between UAVs and the charging pad platform, this, in turn, simplifies landing protocols. The result shows that the charging circuit successfully charged the UAV battery until $12.5 \mathrm{v}$.
\end{abstract}

Keyword: Charging systems; UAV; Landing; Charging platform; Electrical fundamentals 